Arquivos Brasileiros de Educação Física
Brazilian Archives of Physical Education $\mathrm{ABEF}$

\title{
Estudo comparativo de valores morfológicos entre atletas femininas de beach handball de diferentes qualificações esportivas
}

Comparative study of morphological values among female beach handball athletes of different sports qualifications

Estudio comparativo de valores morfológicos entre mujeres playa balonmano atletas de diferentes calificaciones deportivas

\author{
José Ednaldo Alves de Sena' \\ Vanessa Manuela Neves da Silva ${ }^{2}$ \\ Urival Magno Gomes Ferreira ${ }^{3}$
}

\section{Resumo}

O Beach Handball é um esporte em que além de fatores genéticos são exigidas também características morfológicas específicas. Foram avaliados os valores morfológicos do percentual de gordura (\%G) e do índice de massa corporal (IMC) de 32 atletas; 10 de alto rendimento; 11 de rendimento intermediário e 11 de baixo rendimento. Utilizou-se a média, desvio-padrão, mínimo, máximo, ANOVA one-way, teste de comparação múltipla de Scheffé, com nível de significância de $\mathrm{p}<0,05$. Existe diferença $(\mathrm{p}<0,005)$ para: \%G. Os grupos apresentaram em média um IMC eutrófico, com o alto, intermediario e baixo rendimento, apresentando respectivamente $22,74 \mathrm{~kg} / \mathrm{m}^{2}, 23,70 \mathrm{~kg} / \mathrm{m}^{2}$ e $22,01 \mathrm{~kg} / \mathrm{m}^{2}$ com $\% \mathrm{G}$ acima de valores para o beach handball. Ao comparar valores morfológicos entre atletas femininas de beach handball de diferentes qualificações esportivas, não obstante ter havido diferenças significativas entre as mesmas, com a equipe de alto rendimento apresentando melhores escores, em todas, à adequação ao genótipo deve ser ação primordial quando da prescrição dos treinos, afim de que se possa obter o máximo desenvolvimento da performance das atletas. Por fim recomenda-se que os dados aqui apresentados sirvam de subsídios para a seleção de futuros atletas.

Palavras - chave: Morfologia. Desempenho atlético. Beach Handball.

\begin{abstract}
Beach Handball is a sport in which, in addition to genetic factors, specific morphological characteristics are also required. The morphological values of the fat percentage $(\% \mathrm{~F})$ and the body mass index (BMI) of 32 athletes were evaluated; 10 high yield; 11 of intermediate income and 11 of low income. The mean, standard deviation, minimum, maximum, one-way ANOVA, Scheffé's multiple comparison test were used, with a significance level of $p<0.05$. There is a difference $(\mathrm{p}<0.005)$ for:\% $\mathrm{G}$. The groups presented on average an eutrophic BMI, with high, intermediate and low performance, presenting respectively $22.74 \mathrm{~kg} / \mathrm{m} 2,23.70 \mathrm{~kg} / \mathrm{m} 2$ and $22.01 \mathrm{~kg} / \mathrm{m} 2$ with\% $\mathrm{G}$ above values for beach handball. When comparing morphological values between female beach handball athletes of different sporting qualifications, despite significant differences between them, with the high performance team presenting better scores, in all, the adequacy to the genotype should be a primary action when prescribing the results. training, in order to obtain the maximum development of the athletes' performance. Finally, it is recommended that the data presented here serve as subsidies for the selection of future athletes. Keywords: Morphology. Athletic performance. Beach Handball.

1. Secretaria de Educação e Cultura - João Pessoa/PB, Brasil; 2. Faculdade de Enfermagem Nova Esperança, João Pessoa/PB, Brasil. E-mail para correspondência: urival_magno@hotmail.com . Este conteúdo utiliza a Licença Creative Commons Attribution 4.0 International License Open Access. This content is licensed under a Creative Commons attribution-type BY ISSN 2595 - 0096.
\end{abstract}




\section{Resumen}

El balonmano de playa es un deporte en el que, además de los factores genéticos, también se requieren características morfológicas específicas. Se evaluaron los valores morfológicos del porcentaje de grasa (\% F) y el índice de masa corporal (IMC) de 32 atletas; 10 alto rendimiento; 11 de ingresos intermedios y 11 de bajos ingresos. Se utilizó la media, desviación estándar, mínimo, máximo, ANOVA unidireccional, la prueba de comparación múltiple de Scheffé, con un nivel de significancia de $\mathrm{p}<0.05$. Hay una diferencia $(\mathrm{p}<0.005)$ para:\% G. Los grupos presentaron en promedio un IMC eutrófico, con alto, intermedio y bajo rendimiento, presentando respectivamente $22.74 \mathrm{~kg} / \mathrm{m} 2,23.70 \mathrm{~kg} / \mathrm{m} 2$ y $22.01 \mathrm{~kg} / \mathrm{m} 2$ con valores de\% G por encima para el balonmano de playa. Al comparar los valores morfológicos entre las atletas femeninas de balonmano de playa de diferentes calificaciones deportivas, a pesar de las diferencias significativas entre ellas, con el equipo de alto rendimiento presentando mejores puntajes, en general, la adecuación al genotipo debería ser una acción principal al prescribir los resultados. entrenamiento, para obtener el máximo desarrollo del rendimiento de los atletas. Finalmente, se recomienda que los datos presentados aquí sirvan como subsidios para la selección de futuros atletas.

Palabras - clave: Morfología. Rendimiento atlético. Balonmano de playa.

\section{INTRODUÇÃO}

O handebol de areia conhecido como beach handball é um esporte novo que começou a se popularizar durante os últimos anos da década de 90, com torneios e pequenos campeonatos espalhados por diversos países ${ }^{1}$. Atualmente, o beach handball do Brasil figura entre os melhores do mundo. É um esporte que, devendo ser praticado na areia, exige um preparo físico maior das atletas, sendo já observada sua amplitude como prática esportiva, que vai da informalidade ao alto rendimento. Dentro dessa perspectiva é que surge as variáveis morfológicas como aspectos muito importantes para que a atleta possa manter um bom rendimento durante as competições. Assim, como em outras modalidades esportivas, e de forma mais abrangente, o beach handball exige atletas com características morfológicas específicas, as quais influenciarão, direta ou indiretamente, as ações técnicas e táticas desta modalidade. Este fato é contemplado nos estudos de Glaner ${ }^{2}$, Glaner ${ }^{3}$ e Vasques et al. ${ }^{4}$ Nogueira e Fernandes Filho ${ }^{5}$, Profeta ${ }^{6}$, Silva ${ }^{7}$, Dantas et al. ${ }^{8}$. Dessa forma, diante do supra abordado é que se justifica a necessidade de se conhecer e se entender esta nova modalidade, através de sua composição corporal, propiciando relevantes subsídios científicos para que os técnicos possam usá-los no desenvolvimento da performance das atletas, podendo assim solucionar problema deste estudo que é: Existem ações efetivas, moldadas em parâmetros científicos, por parte de treinadores no que consiste a forma de seleção e prescrição de treino de atletas femininas de beach handball de diferentes qualificações esportivas? O objetivo deste trabalho foi 
comparar valores morfológicos entre atletas femininas de beach handball de diferentes qualificações esportivas.

\section{MÉTODOS}

Ética da pesquisa

O presente trabalho de pesquisa foi submetido e aprovado pelo Comitê de Ética da Pesquisa (CEP) da Rede Euroamericana de Motricidade Humana REMH sob o noㅜ 011/2008 e atenderá às Normas Sobre Pesquisa Envolvendo Seres Humanos, que constam na resolução 196/96 do Conselho Nacional de Saúde, de 10 de Outubro de $1996^{9}$. O Termo de Consentimento Livre e Esclarecido segundo as normas para realização de Pesquisa em Seres Humanos e os critérios de Ética em Pesquisa - Resolução 196/96

\section{Caracterização da pesquisa}

A presente pesquisa é caracterizada conforme Thomas e Nelson ${ }^{32}$ como um estudo transversal, descritivo e comparativo.

\section{Amostra do estudo}

A amostra utilizada e escolhida de maneira intencional consistiu de $\mathrm{n}=32$ atletas do sexo feminino, sendo que destas, $(n=10)$ eram atletas de alto rendimento que compunha a Seleção Brasileira adulta feminina $(\mathrm{n}=11)$; atletas de rendimento intermediário jogadoras da seleção paraibana feminina adulta, $(\mathrm{n}=11)$ atletas de baixo rendimento, jogadoras de equipes que disputam torneios de bairro e/ou no máximo municipal, todas aparentemente saudáveis, com idades entre 14 a 42 anos.

\section{Protocolos e instrumentos}

Massa corporal: para mensurar o peso corporal, foi utilizada uma balança antropométrica da marca Filizola, modelo Personal, com capacidade para $180 \mathrm{~kg}$ e com a acuidade de $100 \mathrm{~g} \cdot{ }^{10,11}$.

Estatura: para a medida da estatura, foi utilizado um estadiômetro tipo trena da marca Seca, com $200 \mathrm{~cm}$ de comprimento, com escala de divisão em milímetros. A estatura foi registrada em centímetros, respeitando-se uma casa decimal. ${ }^{10-12}$.

Circunferências: para mensurar a circunferência do braço em contração isométrica máxima e da panturrilha foi utilizada uma fita de medida antropométrica marca Mabis - Modelo Gulick - confeccionada em fibra de vidro, com $150 \mathrm{~cm}$ de comprimento e graduada em milímetros ${ }^{12,13}$.

Dobras cutâneas: As medidas das dobras cutâneas tricipital, supra-iliaca, coxa, subescapular, supra-espinhal e perna foram obtidas através de um adipômetro da marca Sanny - AMB, com precisão de $0,1^{14,15}$.

Diâmetros: O instrumento empregado foi um paquímetro da marca WCS e com divisão de $1 / 1 \mathrm{~mm}$. Diâmetro biepicondiliano. dedos médios para 
delimitar o diâmetro bi-epicondiliano enquanto os dedos indicadores controlavam as hastes do paquímetro para realizar a medição. Diâmetro biepicondiliano $^{15,16}$.

\section{Protocolos das variáveis morfológicas}

Índice de Massa Corporal - IMC: as características morfológicas globais foram delineadas através do IMC - Índice de Massa Corporal, que é a razão entre o peso do indivíduo em quilos e sua altura em metros ao quadrado ${ }^{17}$. Os pontos de cortes do IMC para avaliação do estado nutricional das atletas foram obtidos segundo a classificação da $\mathrm{OMS}^{18}$.

Percentual de gordura: para as atletas adultas, inicialmente estimou-se a densidade corporal, utilizando-se da equação de Jackson, Pollock e Ward citado por $\operatorname{Costa}^{19}$. Para a realização do cálculo do percentual de gordura $(\% G)$ foi utilizada a equação proposta por Siri citada por Guedes $^{20}$. O percentual de gordura para as adolescentes foi estimado a partir da equação de Slaughter et $\mathrm{al}^{21}$ a qual considera o gênero, a raça e o grau de maturação. A classificação desses resultados foi realizada através de pontos de corte propostos por Deurenberg et al. ${ }^{22}$.

Foi utilizado o Software Physical Test versão 6.2 para realização de classificações.

\section{Análise de dados}

Para formar o banco de dados e utilizar as técnicas da estatística descritiva de média (x), desvio-padrão (DP), mínimo, máximo e percentuais, utilizou-se do SPSS (Statistical Package for Social Sciences) versão 16.0. Para observação das técnicas da Estatistica Inferencial, inicialmente, com o intuito de aumentar a potência dos resultados e garantir a confiabilidade dos mesmos, foi aplicado o teste NãoParamétrico de Normalidade, KomogorovSmirnov, o qual presta-se a verificar se os valores médios das variáveis estudadas, apresentam comportamento ajustado a uma Curva Normal (Gaussiana). Em seguida, foi aplicado o teste estatístico ANOVA one-way para analisar e identificar possíveis diferenças estatisticamente significativas e comparações múltiplas pelo teste post hoc de Scheffé com o nível de significância adotado de 5,0\% (p<0,05). Foi usado o Excel 2007, para elaboração de tabelas.

\section{RESULTADOS E DISCUSSÃO}

Analisando-se a tabela 1, pode ser observado que há diferença significativa nos grupos estudados para as variáveis Idade $(\mathrm{p}=0,001)$, Massa corporal $(\mathrm{p}=0,021)$, Estatura $(p=0,016)$ e Percentual de gordura $(\mathrm{p}=0,008)$. Entretanto no IMC não se observou resultado significativo entre as médias destes grupos. 


\section{CONCLUSÃO}

Desta forma, os resultados deste estudo demonstraram que, ao comparar valores morfológicos entre atletas femininas de Beach handball de diferentes qualificações esportivas, não obstante ter havido diferenças significativas entre as mesmas, com a equipe de alto rendimento apresentando melhores escores, em todas, à adequação ao genótipo deve ser ação primordial quando da prescrição dos treinos, afim de que se possa obter o máximo desenvolvimento da performance das atletas. Por fim recomenda-se que os dados aqui apresentados sirvam de subsídios para a seleção de futuros atletas.

\section{REFERÊNCIAS}

1. Belloli L. História do Handebol. [acesso em 2009 maio 23]. Disponível em: http://www.handebolsatc.kit.net.

2. Glaner MF. Morfologia de atletas panamericanos de handebol adulto masculino. [Dissertação de Mestrado], UFSM, Santa Maria, RS, 1996 (a).

3. Glaner MF. Perfil morfológico dos melhores atletas pan-americanos de handebol por posição de jogo. Revista Brasileira de Cineantropometria e Desempenho Humano. 1999; 1(1): 69$81(\mathrm{~b})$.

4. Vasques DG, Antunes PC, Duarte MFS, Lopes AS. Morfologia dos Atletas de handebol masculino de Santa Catarina. R Bras Ci e Mov. 2005; 13 (2): 49-57.

5. Nogueira TN, Menezes LS, Dantas PMS, Fernandes Filho J. Perfil Morfológico e de aptidão física das atletas de Handebol do Esporte Clube Mauá. In: Simpósio Internacional em Treinamento Desportivo,
3. João Pessoa, PB. Anais_ CDROM, 2002.

6. Profeta GW. Caracterização de um perfil somatotípico do Atleta Brasileiro de Handebol, baseado no método Heath e Carter. 1983. [Dissertação]. Universidade Federal de Santa Maria.

7. Silva LHM. Estudo do perfil somatotípico antropométrico de handebolistas universitários das regiões Norte-Nordeste e Centro-Sul do Brasil. 1985. [Dissertação]. Universidade de São Paulo.

8. Dantas PMS et al. Identificação dos perfis dermatoglífico e somatotípico de atletas iniciantes em Handebol feminino da cidade de Natal - RN. FIEP BULLETIN. Foz do Iguaçu. 2003; 73, 46- 50.

9. Brasil. Conselho Nacional de Saúde. Resolução no 196 de 10 de outubro de 1996. Diretrizes e normas regulamentadoras de pesquisas envolvendo seres humanos. Diário Oficial da República Federativa do Brasil, Brasília (DF); 1996 Out 16. [acesso em 2004 Set 20]. Disponível em: http://conselho.saude.gov.br/docs/Reso196 .doc.

10. Guedes DP, Guedes JERP. Manual prático para avaliação em educação física. São Paulo: Manole; 2006.

11. Fernandes Filho J. A prática da avaliação física: testes, medidas e avaliação física em escolares, atletas e academias de ginástica. $2^{\underline{a}}$ ed. Rio de Janeiro: Shape; 2003.

12. Pitanga FJG. Testes, medidas e avaliação em educação física e esportes. São Paulo: Phorte; 2004.

13. Matsudo SMM. Avaliação do idoso: física e funcional. Londrina: MIDIOGRAF; 2000.

14. Petroski EL. (Org.) Antropometria: técnicas e padronizações. Porto Alegre: Palotti; 1999.

15. Fontoura AS, Formentin CM, Abech EA. Guia prático de avaliação física: uma abordagem didática, abrangente e atualizada. São Paulo: Phorte; 2008. 
16. Rocha PECP. Medidas e avaliação em ciências do esporte. Rio de Janeiro: Sprint; 1995.

17. Cronk CE, ROCHE AF. Race and Sexspecific reference data for triceps and subscapular skinfold and weight/stature 2 . The American Journal of Clinical Nutrition. Bethesda. feb; 35(2):347354;1982.

18. Organização Mundial de Saúde - OMS. Physical status: the use and interpretation of anthropometry: report of a WHO expert committee. WHO Technical Report Series n. 854. Genebra; 1995.

19. Costa RF. Composição corporal: teoria e prática. São Paulo: Manole; 2001.

20. Guedes DP. Composição corporal: princípios, técnicas e aplicações. Florianópolis: CEITEC; 1989.

21. Slaughter MH, Lohman TG, Boileau RA, Horswill CA, Stillman RJ, Vanloan MD, et al. Skinfold equations for estimation of body fatness in children and youth. Hum Biol 1988; 60:709-23.

22. Deurenberg P, Pieters JJ, Hautvast JG. The assessment of the body fat percentage by skinfold thickness measurements in childhood and young adolescence. British Journal of Nutrition. 1990; 63: 293-303.

23. Matsudo, Matsudo, VCR. Testes em ciências do esporte . São Paulo, Centro de Estudos do Laboratório de aptidão Física de São Caetano do Sul,1991.

24. Fróes MQ, Pelozin F, Vasques DG, Pacheco RL. Fernandes LL. Comparação antropométrica entre atletas de handebol da UFSC com atletas de alto nivel e seleção brasileira. Revista Eletrônica de Extensão, 4(5); 2007.

25. Anjos LA. Índice de massa corporal (massa corporal/estatura ${ }^{2}$ ) como indicador do estado nutricional de adultos: revisão da literatura. Rev. Saúde Pública, São Paulo, 1992; 26(6).

26. Must A, Dallal GE, Dietz WH. Reference data for obesity: 85th and 95th percentiles of body mass index (wt/ht2) and triceps skinfold thickness. Am J Clin Nutr; 53:839-46;1991.
HEALTH

ORGANIZATION. The world health report 1997. Geneva; 1997.

28. Foss ML, Keteyian SJF. Bases fisiológicas do exercício e do esporte. $6^{\mathrm{a}} \mathrm{ed}$. Rio de Janeiro: Guanabara Koogan; 2000.

29. Colares LGT. Estudo dietético e antropométrico de atletas competitivos de handebol. 1994. [Dissertação]. Universidade Federal do Rio de Janeiro. 30. Fróes MQ, Pelozin F, Vasques DG, Pacheco RL, Fernandes LL. Comparação antropométrica entre atletas de handebol da UFSC com atletas de alto nível e seleção brasileira. Revista Eletrônica de Extensão. 4(5); 2007.

31. Lohman TG. Advances in body composition assessment. Human Kinetics Publishers, Champaing, IL, 1992.MALINA, R.M. Bioeletrical methods for estimating body composition: An overview and discussion. Human Biology.59;329-335; 1987.

32. Thomas JR, Nelson JK. Métodos de pesquisa em atividade física. $5^{\mathrm{a}}$ ed. Porto Alegre: Artmed; 2007.

Informações do artigo /

Information of this article:

Recebido: 11/04/2020

Aprovado: 21/08/2020

Publicado: 28/12/2020

Received: 11/04/2020

Approved: 21/08/2020

Published: 28/12/2020

Como citar esse artigo / How to cite this article:

Sena, J. E. A.; Silva, V. M. N.; Ferreira, U. M. G. Estudo comparativo de valores morfológicos entre atletas femininas de beach handball de diferentes qualificações esportivas. Arq. Bras. Ed. Fís., Tocantinópolis, v. 3, n. 2, Ago./Dez., p. 27 - 32, 2020. 